\title{
A NOTE ON THE ZEROS OF THE ZETA-FUNCTION OF RIEMANN
}

\author{
AleKSANDAR IVIĆ
}

\begin{abstract}
It is shown explicitly how the sign of Hardy's function $Z(t)$ depends on the parity of the zero-counting function $N(T)$. Two existing definitions of this function are analyzed, and some related problems are discussed.
\end{abstract}

\section{INTRODUCTION}

Let the Riemann zeta-function be, as usual,

$$
\zeta(s)=\sum_{n=1}^{\infty} n^{-s} \quad(\Re s>1) .
$$

For $\Re s \leqslant 1$ one defines $\zeta(s)$ by analytic continuation (see the monographs of H.M. Edwards [1], the author [2], [3] and E.C. Titchmarsh [9] for an extensive account on $\zeta(s))$.

Hardy's function (see the author's monograph [5] for its properties) is

$$
Z(t):=\zeta\left(\frac{1}{2}+i t\right)\left(\chi\left(\frac{1}{2}+i t\right)\right)^{-1 / 2}, \zeta(s)=\chi(s) \zeta(1-s)
$$

so that

$$
\chi(s)=\frac{\Gamma\left(\frac{1}{2}(1-s)\right)}{\Gamma\left(\frac{1}{2} s\right)} \pi^{s-1 / 2} .
$$

The relation $\zeta(s)=\chi(s) \zeta(1-s)$ holds $\forall s \in \mathbb{C}$. It was proved first by B. Riemann in 1859 , and this is the well-known functional equation for the zeta-function. In

1991 Mathematics Subject Classification. 11M06.

Key words and phrases. Riemann zeta-function, Hardy's function $Z(t)$, definition of $N(T)$, Riemann hypothesis. 
1914 G.H. Hardy introduced $Z(t)$ to prove that there are infinitely many zeros of $\zeta(s)$ on the so-called "critical line" $\Re s=1 / 2$.

From $(1.1),(1.2)$ and $\chi(s) \chi(1-s)=1$ follows that $Z(t)$ is a smooth, even, real-valued function of the real variable $t$, for which $|Z(t)|=\left|\zeta\left(\frac{1}{2}+i t\right)\right|$. Further we have

$$
\left(\chi\left(\frac{1}{2}+i t\right)\right)^{-1 / 2}=\pi^{-i t / 2} \frac{\Gamma^{1 / 2}\left(\frac{1}{4}+\frac{1}{2} i t\right)}{\Gamma^{1 / 2}\left(\frac{1}{4}-\frac{1}{2} i t\right)}=\pi^{-i t / 2} \frac{\Gamma\left(\frac{1}{4}+\frac{1}{2} i t\right)}{\left|\Gamma\left(\frac{1}{4}+\frac{1}{2} i t\right)\right|}:=\mathrm{e}^{i \theta(t)},
$$

say. Thus (1.3) implies that, for $t \in \mathbb{R}$,

$$
\begin{aligned}
\theta(t) & =-\frac{1}{2 i} \log \chi\left(\frac{1}{2}+i t\right)=\frac{1}{i}\left\{\log \Gamma\left(\frac{1}{4}+\frac{1}{2} i t\right)-\log \left|\Gamma\left(\frac{1}{4}+\frac{1}{2} i t\right)\right|\right\}-\frac{1}{2} t \log \pi \\
& =\arg \Gamma\left(\frac{1}{4}+\frac{1}{2} i t\right)-\frac{1}{2} t \log \pi=\Im\left\{\log \Gamma\left(\frac{1}{4}+\frac{1}{2} i t\right)\right\}-\frac{1}{2} t \log \pi \in \mathbb{R} .
\end{aligned}
$$

The problem of the distribution of complex zeta zeros $\rho_{n}=\beta_{n}+i \gamma_{n}$ is a fundamental one in zeta-function theory. In view of the functional equation and $\overline{\zeta(s)}=\zeta(\bar{s})$, we may assume without loss of generality that $\frac{1}{2} \leqslant \beta_{n}<1$ (since it is elementary that there are no zeros in the region $\Re s \geqslant 1$ ) and $\gamma_{n}>0$. Here the ordinates of zeros are ordered as $0<\gamma_{1} \leqslant \gamma_{2} \leqslant \gamma_{3} \leqslant \cdots$, with ordinates belonging to multiple zeros (if any) being considered as different. All known zeros are simple, and the first three in the upper complex half-plane are approximately

$$
\frac{1}{2}+i 14.134725 \ldots, \frac{1}{2}+i 21.022039 \ldots, \frac{1}{2}+i 25.010857 \ldots
$$

For recent results on zeta-zeros, see e.g., S. Wedeniwski [10].

If $\gamma(>0)$ denotes generic ordinates of zeta zeros, then the integer-valued function

$$
N(T):=\sum_{0<\gamma \leqslant T} 1
$$

counts the number of $\gamma$ 's not exceeding $T$ (with multiplicities included), and is therefore well-defined for any $T>0$. This is the definition from E.C. Titchmarsh [9], p. 211. There is another definition of $N(T)$, namely

$$
\begin{aligned}
& N(T):=\sum_{0<\gamma<T} 1 \quad(T \neq \gamma) \\
& N(T):=\frac{1}{2}(N(T-0)+N(T+0)) \quad(T=\gamma) .
\end{aligned}
$$

This definition is already in the title of A. Selberg's paper [8], but I am not certain in which works the above definitions appeared first. Unless $T=\gamma$ both definitions 
of $N(T)$ are equal, and in the applications which I have seen so far it does not matter which one of them is used. However, if $N(\gamma-0)$ and $N(\gamma+0)$ are of different parity, then according to Selberg's definition $N(\gamma)$ is of the form $2 k-\frac{1}{2}, k \in \mathbb{N}$, and thus not an integer. We shall see later that this fact is of some significance.

A version of the well known Riemann - von Mangoldt formula for $N(T)$ (see p. 212 of E.C. Titchmarsh [9] and equation (1.45) of [5]) asserts that

$$
N(T)=\frac{1}{\pi} \theta(T)+1+S(T) \quad(T \neq \gamma)
$$

with

$$
S(T):=\frac{1}{\pi} \arg \zeta\left(\frac{1}{2}+i T\right) \quad(T \neq \gamma)
$$

Here $\theta(T)$ is as in (1.4), while $\arg \zeta\left(\frac{1}{2}+i T\right)$ is evaluated by continuous variation starting from $\arg \zeta(2)=0$ and proceeding along straight lines, first up to $2+i T$ and then to $1 / 2+i T$, assuming that $T$ is not an ordinate of a zeta zero. If $T$ is an ordinate of a zero, then we set $S(T)=S(T+0)$. Again, this is according to [9], while Selberg [8] defines

$$
S(T)=\frac{1}{2}(S(T-0)+S(T+0)) \quad(T=\gamma)
$$

which is in tune with (1.6). From (1.7) and (1.8) it is not difficult to deduce that

$$
N(T)=\frac{T}{2 \pi} \log \left(\frac{T}{2 \pi}\right)-\frac{T}{2 \pi}+\frac{7}{8}+S(T)+O\left(\frac{1}{T}\right)
$$

where the $O$-term is a continuous function of $T$, and $S(T)=O(\log T)$.

\section{Connection Between $Z(t)$ And $N(t)$}

From $|Z(t)|=\left|\zeta\left(\frac{1}{2}+i t\right)\right|$ and the fact that $Z(t)$ is real it follows that either $Z(t)=\left|\zeta\left(\frac{1}{2}+i t\right)\right|$ or $Z(t)=-\left|\zeta\left(\frac{1}{2}+i t\right)\right|$, but it is not clear which of these relations holds. A new formula s presented here, which settles this question by connecting the functions $Z(t)$ and $N(T)$. This is

THEOREM 1. If $t$ is not the ordinate of any zeta-zero $\beta_{n}+i \gamma_{n}$, then

$$
Z(t)=(-1)^{N(t)+1}\left|\zeta\left(\frac{1}{2}+i t\right)\right| \quad(t>0) .
$$

Theorem 1 provides an unconditional connection between the sign of $Z(t)$ and the parity of the zero-counting function $N(t)$. We assume that $N(T)$ is defined by (1.5). We start from (1.7), which gives

$$
\theta(T)=\pi N(T)-\pi S(T)-\pi \quad\left(T \neq \gamma_{n}\right)
$$


where $T$ is not an ordinate of any zeta zero $\beta_{n}+i \gamma_{n}$. We record the explicit representation of $\theta(t)$ (see (1.21) and (1.22) of [5]), which follows from (1.4) and Stirling's classical formula for the gamma-function. This is

$$
\theta(t)=\frac{t}{2} \log \frac{t}{2 \pi}-\frac{t}{2}-\frac{\pi}{8}+\Delta(t) \quad(t>0)
$$

Here

$$
\Delta(t):=\frac{t}{4} \log \left(1+\frac{1}{4 t^{2}}\right)+\frac{1}{4} \arctan \frac{1}{2 t}+\frac{t}{2} \int_{0}^{\infty} \frac{\psi(u)}{\left(u+\frac{1}{4}\right)^{2}+\left(\frac{1}{2} t\right)^{2}} \mathrm{~d} u
$$

with

$$
\psi(x):=x-[x]-\frac{1}{2}=-\sum_{n=1}^{\infty} \frac{\sin (2 n \pi x)}{n \pi} \quad(x \notin \mathbb{Z}) .
$$

We have the approximation (see e.g., p. 120 of H.M. Edwards [1])

$$
\theta(t)=\frac{t}{2} \log \frac{t}{2 \pi}-\frac{t}{2}-\frac{\pi}{8}+\frac{1}{48 t}+\frac{7}{5760 t^{3}}+O\left(\frac{1}{t^{5}}\right)
$$

For any complex number $z$ one has $z=|z| \mathrm{e}^{i \arg z}$. Hence from the defining relation (1.1) and (1.3) it follows that

$$
Z(t)=\zeta\left(\frac{1}{2}+i t\right) \mathrm{e}^{i \theta(t)}=\left|\zeta\left(\frac{1}{2}+i t\right)\right| \mathrm{e}^{i \arg \zeta\left(\frac{1}{2}+i t\right)+i \theta(t)}
$$

We have, on using (1.8) and (2.2),

$$
i \arg \zeta\left(\frac{1}{2}+i t\right)+i \theta(t)=i \pi S(t)+i \pi N(t)-\pi i S(t)-i \pi=i \pi N(t)-i \pi .
$$

But since $\mathrm{e}^{ \pm \pi i}=-1$, we obtain from $(2.2)$

$$
Z(t)=(-1)^{N(t)+1}\left|\zeta\left(\frac{1}{2}+i t\right)\right| \quad(t \neq \gamma)
$$

as asserted in Theorem 1. Clearly there is no restriction on $t$, thus (2.1) holds for any $t>0$ such that $t \neq \gamma$, i.e., if $t$ is not an ordinate of any zeta zero. In the course of the proof we used (1.5). However, in the case when $t \neq \gamma$, which is assumed in the formulation of Theorem 1, both definitions of $N(T)$ in (1.5) and (1.6) coincide. Therefore Theorem 1 remains (unconditionally) true regardless of which definition of $N(T)$ one uses. 


\section{Discussion of the two Definitions of $N(T)$}

If the famous Riemann Hypothesis $(\mathrm{RH}$, all complex zeros of $\zeta(s)$ satisfy $\Re s=$ $1 / 2$ ) is true, then the (real) zeros of $Z(t)$ correspond to the zeros $1 / 2+i t$ of $\zeta(s)$. In this case both sides of (2.1) vanish, so Theorem 1 holds in this case for all $t>0$, regardless of which definition of $N(T)$ is used.

Suppose now that the $\mathrm{RH}$ fails. Then there exist $\beta, \gamma \in \mathbb{R}$ such that $1 / 2<$ $\beta<1, \gamma>0$ and $\zeta(\beta+i \gamma)=\zeta(1-\beta+i \gamma)=0$. Moreover, the functional equation $\zeta(s)=\chi(s) \zeta(1-s)$ implies that both $\beta+i \gamma$ and $1-\beta+i \gamma$ have the same multiplicity. Recall that if $r=m(\rho)$ denotes the multiplicity of the complex zero $\rho=\beta+i \gamma$ of $\zeta(s)$, then

$$
\zeta(\rho)=\zeta^{\prime}(\rho)=\ldots=\zeta^{(r-1)}(\rho)=0, \text { but } \zeta^{(r)}(\rho) \neq 0
$$

Furthermore a zero $\rho$ is simple if $m(\rho)=1$, namely if $\zeta(\rho)=0$ but $\zeta^{\prime}(\rho) \neq 0$. The problem of evaluating the multiplicities of zeta zeros is a deep one (see the author's papers [4], [6], and A.A. Karatsuba [7]). Suppose we use the definition of $N(T)$ given by (1.5). Note that by continuity $Z(\gamma)=Z(\gamma-0)$, and $(2.1)$ holds for $t=\gamma-\varepsilon$, and sufficiently small $\varepsilon>0$. The contribution of $N(\gamma-0)$ differs from that of $N(\gamma)$ by the contribution of $S:=\sum_{j=1}^{k} 2 m\left(\beta_{j}+i \gamma\right)$, where $\frac{1}{2}<\beta_{1}<\cdots<\beta_{k}<1$ and $\zeta\left(\beta_{j}+i \gamma\right)=0(j=1, \ldots, k)$. Here we assumed that the $\beta_{j}$ 's are the real parts of all different values of $\frac{1}{2}<\beta<1$ such that $\zeta(\beta+i \gamma)=0$. Whatever the value of $k$ is, the sum $S$ is an even natural number $2 K, K=K(k)$, hence $N(\gamma)=N(\gamma-0)+2 K$. Then we have

$$
\begin{aligned}
Z(\gamma) & =Z(\gamma-0)=(-1)^{N(\gamma-0)+1}\left|\zeta\left(\frac{1}{2}+i(\gamma-0)\right)\right| \\
& =(-1)^{N(\gamma)-2 K+1}\left|\zeta\left(\frac{1}{2}+i \gamma\right)\right|=(-1)^{N(\gamma)+1}\left|\zeta\left(\frac{1}{2}+i \gamma\right)\right| .
\end{aligned}
$$

This shows that (2.1) holds if $t=\gamma$, and the same conclusion holds if we use $Z(\gamma)=Z(\gamma+0)$

However, if we use the second definition of $N(T)$ in (1.6), then (2.1) does not have to hold for $t=\gamma$, no matter how we define $Z(\gamma)$ by a limit process. Namely, as already noted, if $N(\gamma-0)$ and $N(\gamma+0)$ are of different parity, then according to Selberg's definition $N(\gamma)$ is of the form $2 k-\frac{1}{2}, k \in \mathbb{N}$, hence $(-1)^{N(\gamma)+1}$ is neither +1 nor -1 . This happens e.g., if $N(\gamma-0)$ is even, but $\frac{1}{2}+i \gamma$ is a simple zero, and there are no other zeta zeros $\rho$ with $\Im \rho=\gamma$. We see that, in the context of Theorem 1, it does matter which definition of $N(T)$ is used. If we use the definition of $N(T)$ in (1.5), then actually (2.1) holds for all $t>0$ unconditionally.

In fact, the argument of the previous section provides another proof that (2.1) holds unconditionally if (1.5) is used. In view of (1.2) one has

$$
Z(0)=\chi^{-1 / 2}\left(\frac{1}{2}\right) \zeta\left(\frac{1}{2}\right)=\zeta\left(\frac{1}{2}\right)<0,
$$


which establishes $(2.1)$ for $0<t \leqslant \gamma_{1}$. We can then easily verify that (2.1) holds for $t<10^{13}$, e.g., when $t$ does not exceed the ordinate of the largest known zero (see [10]). Since all known zeros are simple and satisfy the $\mathrm{RH}, N(T)$ jumps by unity at each $\gamma$, so (2.1) holds. Let $\gamma_{N}$ be the largest known ordinate of a zetazero, so that $(2.1)$ holds for $t \leqslant \gamma_{N}$. Since $\frac{1}{2}+i \gamma_{N}$ is a simple zero, then $Z(t)$ changes sign at $\gamma_{N}$, so $(2.1)$ holds also in $\left[\gamma_{N}, \gamma_{N+1}\right)$. At $t=\gamma_{N+1}$ two cases are possible. The first case is when $\frac{1}{2}+i \gamma_{N+1}$ is a zeta-zero, and there is no $\beta>\frac{1}{2}$ such that $\zeta\left(\beta+i \gamma_{N+1}\right)=0$. In that case $N(t)$ jumps at $t=\gamma_{N+1}$ by $m\left(\frac{1}{2}+i \gamma_{N+1}\right)$. If this number is odd, then $Z(t)$ changes sign at $\gamma_{N+1}$ and (2.1) is true for $\gamma_{N+1} \leqslant t<\gamma_{N+2}$. If this number is even, then $Z(t)$ maintains its sign in the same interval, and again (2.1) is true for $\gamma_{N+1} \leqslant t<\gamma_{N+2}$. The same is true if there are zeros $\rho$ off the critical with this $\gamma_{N+1}$. The contribution of these zeros to $N\left(\gamma_{N+1}\right)$, as seen in the previous section, is an even number. In the second case, when $\zeta\left(\frac{1}{2}+i \gamma_{N+1}\right) \neq 0$, but there is a $\beta\left(>\frac{1}{2}\right)$ such that $\zeta\left(\beta+i \gamma_{N+1}\right)=0$ is similar. The contribution to $N\left(\gamma_{N+1}\right)$ is an even number, and so it does not affect the sign of $(-1)^{N(t)+1}$. In any case $(2.1)$ is verified for $\gamma_{N+1} \leqslant t<\gamma_{N+2}$, and the proof of (2.1) may be given inductively.

Corollary 1. Suppose $Z\left(t_{1}\right) \neq 0, Z\left(t_{2}\right) \neq 0$. Then $Z\left(t_{1}\right)$ and $Z\left(t_{2}\right)$ are both of the same sign if and only if $N\left(t_{1}\right)$ and $N\left(t_{2}\right)$ are of the same parity, provided that $N(T)$ is defined by (1.5).

This follows from (2.1), since we have

$$
\begin{aligned}
Z\left(t_{1}\right) Z\left(t_{2}\right) & =(-1)^{N\left(t_{1}\right)+1}(-1)^{N\left(t_{2}\right)+1}\left|\zeta\left(\frac{1}{2}+i t_{1}\right)\right|\left|\zeta\left(\frac{1}{2}+i t_{2}\right)\right| \\
& =(-1)^{N\left(t_{1}\right)+N\left(t_{2}\right)}\left|\zeta\left(\frac{1}{2}+i t_{1}\right) \zeta\left(\frac{1}{2}+i t_{2}\right)\right| .
\end{aligned}
$$

Thus $Z\left(t_{1}\right)$ and $Z\left(t_{2}\right)$ are both of the same sign iff the left-hand side of $(2.5)$ is positive, and this happens iff $N\left(t_{1}\right)$ and $N\left(t_{2}\right)$ are of the same parity.

Finally, one may consider the following problems. First, for how many $\gamma(\leqslant T)$ the function $N(\gamma)$ is even? Here the definition (1.5) is understood. Does one have

$$
\sum_{0<\gamma \leqslant T, N(\gamma)=2 k} 1 \sim \frac{1}{2} N(T) \quad(T \rightarrow \infty) ?
$$

This is certainly true if the conjecture that all zeros $\rho$ are simple is true. This, however, seems to lie quite deep. The conjectures $R H$ is true and all zeros are simple seem independent of one another. As far as it is known both conjectures could be true, or false, or one true and the other one false.

In the case when $\mathrm{RH}$ fails, it seems of interest to define, for a given $\gamma(>0)$,

$$
A(\gamma):=\sum_{\frac{1}{2}<\beta<1, \zeta(\beta+i \gamma)=\zeta\left(\frac{1}{2}+i \gamma\right)=0} 1
$$


It is clear that

$$
0 \leqslant A(\gamma) \leqslant N\left(\gamma+\frac{1}{2}\right)-N\left(\gamma-\frac{1}{2}\right) \ll \log \gamma
$$

It is reasonable to expect that $A(\gamma)=0$ for almost all $\gamma$, but this is not easy to prove.

\section{REFERENCES}

[1] H.M. Edwards, Riemann's Zeta Function, Academic Press, New York, 1974.

[2] A. Ivić, The Riemann zeta-function, John Wiley \& Sons, New York, 1985 (reissue, Dover, Mineola, New York, 2003).

[3] A. Ivić, Mean values of the Riemann zeta function, Tata Institute of Fund. Research, LN's 82, (distributed by Springer Verlag, Berlin etc.), Bombay, 1991. To be found online at www.math.tifr.res.in/ publ/ln/tifr82.pdf

[4] A. Ivić, On the multiplicity of zeros of the zeta-function, Bulletin CXVIII de l'Académie Serbe des Sciences et des Arts - 1999, Classe des Sciences mathématiques et naturelles, Sciences mathématiques No. 24, pp. 119-131.

[5] A. Ivić, The theory of Hardy's Z-function, Cambridge University Press, Cambridge, 2013, 245 pp.

[6] A. Ivić, On the multiplicites of zeros of $\zeta(s)$ and its values over short intervals, Journal of Number Theory 185(2018), 65-79.

[7] A.A. Karatsuba, Zero multiplicity and lower bound estimates of $|\zeta(s)|$, Funct. Approx. Comment. Math. 35(2006), 195-207.

[8] A. Selberg, On the remainder in the formula for $N(T)$, the number of zeros of $\zeta(s)$ in the strip $0<\gamma<T$, Avhandliger Norske Videnkaps Akad. Oslo. I. Mat.-Naturv. Klasse, 1944, (1944). no. 1, 27 pp, also in A. Selberg, Collected Works I, Springer, Berlin etc., 1989.

[9] E.C. Titchmarsh, The theory of the Riemann zeta-function, 2nd ed. edited by D.R. HeathBrown, Clarendon Press, Oxford, 1986.

[10] S. Wedeniwski, Results connected with the first 100 billion zeros of the Riemann zeta function, 2002, at http://piologie.net/math/zeta.result.100billion.zeros.html

Aleksandar Ivić, Serbian Academy of Science and Arts, Knez Mihailova 35, 11000 Beograd, SERbia

E-mail address:

aleksandar.ivic@rgf.bg.ac.rs, aivic_2000@yahoo.com 